OPEN ACCESS

Edited by:

Alessia Celeghin

University of Turin, Italy

Reviewed by:

Henry Railo,

University of Turku, Finland

Ziad M. Hafed

University of Tübingen, Germany

*Correspondence:

Katsuei Shibuk

shibuki@bri.niigata-u.ac.jp

Specialty section:

This article was submitted to

Neurorehabilitation,

a section of the journal

Frontiers in Neurology

Received: 31 July 2019

Accepted: 20 January 2020

Published: 07 February 2020

Citation:

Shibuki K, Wakui I, Fujimura T,

Tomikawa M and Hasegawa S (2020)

Rapid Recovery From Cortical

Blindness Caused by an Old Cerebral Infarction. Front. Neurol. 11:69. doi: 10.3389/fneur.2020.00069

\section{Rapid Recovery From Cortical Blindness Caused by an Old Cerebral Infarction}

\author{
Katsuei Shibuki ${ }^{1,2 *}$, Ichiro Wakui ${ }^{3}$, Takeo Fujimura ${ }^{3}$, Masaru Tomikawa ${ }^{4}$ and \\ Shin Hasegawa ${ }^{3}$
}

${ }^{1}$ Department of Clinical Laboratory, Kashiwazaki General Hospital and Medical Center, Kashiwazaki, Japan, ${ }^{2}$ Brain Research Institute, Niigata University, Niigata, Japan, ${ }^{3}$ Department of Internal Medicine, Kashiwazaki General Hospital and Medical Center, Kashiwazaki, Japan, ${ }^{4}$ Department of Neurosurgery, Kashiwazaki General Hospital and Medical Center, Kashiwazaki, Japan

When the primary visual cortex (V1) is damaged, cortical blindness results. However, visual information obtained from the superior colliculus (SC) or direct thalamic afferents to higher visual cortices produces unconscious visual functions called blindsight. Alarming visual stimuli suggesting the approach of a predator are known to trigger escape behaviors via visual information mediated by the SC and amygdala in small animals, and salient and dynamic visual stimuli also produce some conscious visual experience even in patients with blindsight. Fresh cortical blindness sometimes recovers spontaneously in patients with fresh cerebral damages, and recovery can be accelerated by early rehabilitation. However, the mechanisms underlying recovery are not well-known. We analyzed a patient with cortical blindness caused by an old cerebral infarction. After repeated presentation of alarming visual stimuli, the ability to detect visual stimuli in the impaired visual field showed behavioral short-term improvement (STI) within a few minutes. Repeated behavioral STI induction was followed by behavioral long-term improvement (LTI) lasting more than several days. After behavioral LTI, the patient partially recovered the ability to read letters presented in the impaired visual field. The behavioral STI experiment, which can be performed within 10 min, may serve as a clinical screening test for anticipating recovery from cortical blindness.

Keywords: blindsight, visual cortex, superior colliculus, short-term potentiation, long-term potentiation, amygdala, looming stimulus

\section{INTRODUCTION}

Visual information mediated via the primary visual cortex $(\mathrm{V} 1)$ is required for conscious sight $(1,2)$. When the V1 is damaged, the visual fields opposite to the damaged V1 are impaired, a condition known as cortical blindness (3-5). However, patients with cortical blindness have unconscious visual functions called blindsight $(6,7)$, possibly through visual information obtained from the superior colliculus (SC), which in turn projects to the amygdala (8-10) and the higher visual cortex (11-14). Fresh cortical blindness sometimes spontaneously recovers (15), and the recovery is facilitated by early rehabilitation $(16,17)$. Possibly, transient malfunctions of cortical areas surrounding the infarction may recover spontaneously, or neural plasticity in the remaining cortical areas may partly compensate for the impaired visual functions (18-20). Another mechanism for 


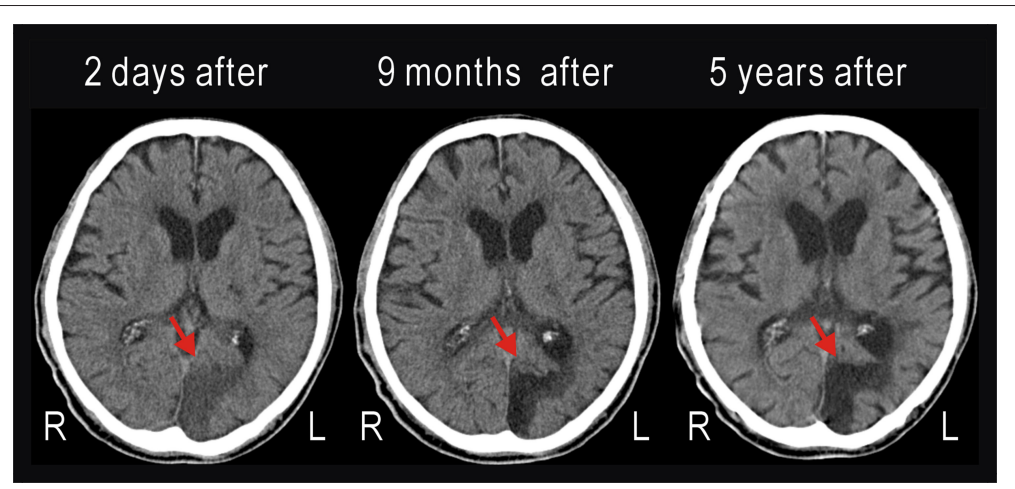

FIGURE 1 | Computed tomography (CT) images taken 2 days, 9 months, and 5 years after cerebral infarction had occurred in the left occipital lobe of the patient. The infarction (red arrow) was unchanged during 5 years.

recovery is that blindsight is converted to conscious sight by neural synchronization between a neuronal group involved in blindsight, and another neuronal group involved in conscious sight $(21,22)$. If so, recovery from blindsight may be possible even in a patient with an old infarction, as long as some latent neural pathways between neurons involved in blindsight and those involved in conscious sight remain.

We investigated a patient with cortical blindness caused by an old cerebral infarction. Repeated presentation of alarming visual stimuli such looming discs $(23,24)$ induced behavioral short-term improvement (STI) of visual stimulus detection in the impaired right visual field. After repeated behavioral STI induction, behavioral long-term improvement (LTI) of visual stimulus detection, lasting for more than several days, was observed. Once behavioral LTI was induced, the patient showed partial recovery in the ability to read letters presented in the impaired right visual field. Although cortical blindness in the present case was caused by an old cerebral infarction, we unexpectedly observed rapid recovery process from cortical blindness as behavioral STI and LTI of visual stimulus detection for the first time.

\section{METHODS}

\section{Patient}

We investigated an 87-year-old male patient, who suffered from diabetes mellitus and a left occipital lobe infarction occurred 5 years ago (Figure 1). He was admitted to Kashiwazaki General Hospital and Medical Center for glycemic control using hypodermic insulin injection. He was also diagnosed with mild dementia based on Mini Mental State Examination. He could read Japanese Hiragana letters with 24 points (approximately 7 $\times 7 \mathrm{~mm}$ ) shown at $600 \mathrm{~mm}$ away from him.

\section{Estimation of Visual Function}

The examiner faced the patient directly at a distance of $600 \mathrm{~mm}$. A tablet computer (Surface Pro 6, Microsoft) was held directly to the left or right of the examiner's face (Figure 2). Various videos created using PowerPoint were presented to the patient. The visual stimuli appeared for $0.5 \mathrm{~s}$ in a $188 \mathrm{~mm}$ diameter circular range (black circle in Figure 2), the center of which was approximately $200 \mathrm{~mm}$ (18.4 $4^{\circ}$ of visual angle) away from the fixation point between both eyes of the examiner (red point in Figure 2). When the examiner judged that the patient was looking at the fixation point, the examiner clicked on a small wireless mouse (M-CC2BRSWH, Elecom, Osaka, Japan). The click triggered an animation of the power point file and various visual stimuli appeared for $0.5 \mathrm{~s}$. As soon as visual stimuli were presented, the patient was to report vocally that it was presented, or report a particular property of the stimuli. The mouse was held in a position invisible to the patient, and the operation of the mouse produced almost no sound. Therefore, the examiner could judge that the patient had seen the visual stimuli if the vocal report occurred immediately after clicking the mouse. When examining the control left visual field, the visual stimulus was shown in the symmetrical position.

\section{Visual Stimuli}

The following visual stimuli were used for testing. Static discs: five black disks were shown for $0.5 \mathrm{~s}$ (Supplementary Video 1); $400 \%$ looming/moving disc: a $47 \mathrm{~mm}$ diameter black disc with complicated movements, enlarging to $188 \mathrm{~mm}$ in diameter for $0.5 \mathrm{~s}$ (Supplementary Video 2); $400 \%$ looming disc: a $47 \mathrm{~mm}$ diameter black disc with no movement, enlarging to $188 \mathrm{~mm}$ in diameter for $0.5 \mathrm{~s}$ (Supplementary Video 3); 150\% looming disc: a $125 \mathrm{~mm}$ diameter black disc with no movement, enlarging to $188 \mathrm{~mm}$ in diameter for $0.5 \mathrm{~s}$ (Supplementary Video 4); slowly appearing disc: a $188 \mathrm{~mm}$ diameter disk changing in color from white to black for $0.5 \mathrm{~s}$ (Supplementary Video 5); suddenly appearing disc: a $188 \mathrm{~mm}$ diameter black disk suddenly appearing, and changing in color from black to white for $0.5 \mathrm{~s}$ (Supplementary Video 6); moving gratings: $20 \mathrm{~mm}$ wide vertical stripes with $40 \mathrm{~mm}$ wide intervals were shown within a $188 \mathrm{~mm}$ diameter circular window, and moved to the right or left by $40 \mathrm{~mm}$ for $0.5 \mathrm{~s}$ (Supplementary Video 7); random letters: one of 46 Japanese Hiragana letters was randomly selected, and shown with a size of 500 points for $0.5 \mathrm{~s}$ (Supplementary Video 8). 


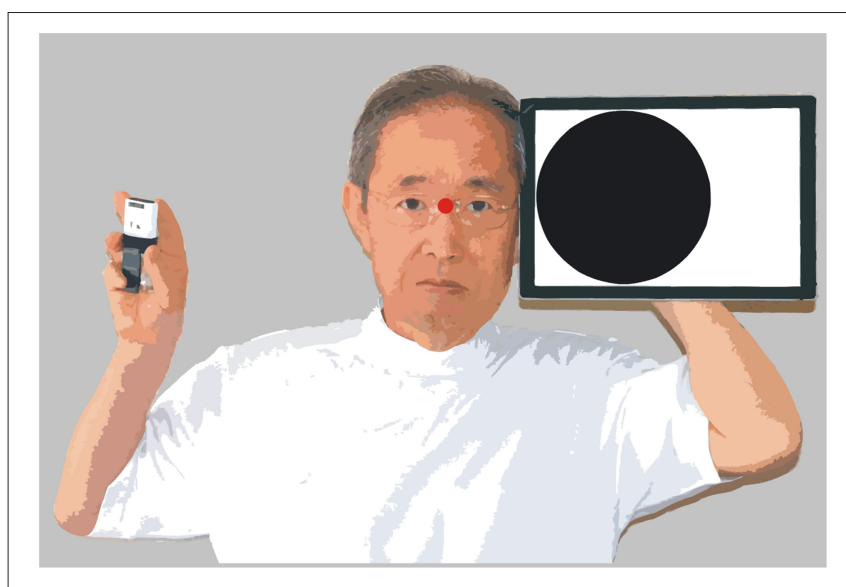

FIGURE 2 | Method for estimating visual functions.

\section{Statistical Analyses}

Statistical significance was evaluated using Pearson's $\chi^{2}$-test, using Easy R, a free software tool for statistical analysis (25). Correction for multiple comparisons was not carried out, as the original $P$-values $\left(1.1 \times 10^{-5} \sim 3.6 \times 10^{-9}\right)$ were sufficiently small.

\section{RESULTS}

\section{Effective Visual Stimuli for the Impaired Right Visual Field}

To confirm the extent of cortical blindness, various static visual stimuli (e.g., static discs, Supplementary Video 1) were shown. The patient could not report stimulus presentation in the impaired right visual field, although the same visual stimuli presented in the control left visual field were noticed with no failures. Next, we presented various dynamic and alarming stimuli in the impaired right visual field. Looming stimuli can be alarming for humans, since the stimuli suggest the presence of rapidly moving objects that may collide with the face. We found that the patient could sometimes notice the presentation of a 400\% looming/moving disc (Supplementary Video 2). He reported that something like a black shadow appeared in the impaired right visual field, as reported previously (26).

\section{Quantitative Estimation of Stimulus Detection}

We found that the patient began to notice the presentation of the static discs in some trials. We used a $400 \%$ looming disc (Supplementary Video 3) and a 150\% looming disc (Supplementary Video 4), and counted the number of trials with successful detection for 10 trials. As a control stimulus, a slowly appearing disc was used (Supplementary Video 5), since this stimulus had been relatively undetectable. These visual stimuli were presented in the impaired right and control left visual fields 10 times, daily for 3 days (Figure 3A, Supplementary Table 1). The results over the 3 days indicated that the $400 \%$ looming disc and 150\% looming disc were significantly better noticed compared with the slowly appearing disc $\left(P<1.1 \times 10^{-5}\right.$ and
$P<7.4 \times 10^{-7}$, respectively). In the control left visual field, the three stimuli were detected in all 30 trials.

\section{Behavioral STI of Visual Stimulus Detection}

While testing the patient, the slowly appearing disc, which had been hardly noticed originally, began to be noticed more frequently. Therefore, the slowly appearing disc was presented in the impaired right visual field for 10 trials followed by control presentation of a suddenly appearing disc (Supplementary Video 6) for other 10 control trials. This session was repeated 3 times (S1-S3) daily for 3 consecutive days (Figure 3B, Supplementary Table 1). The detection probability of the slowly appearing disc was clearly higher in the second and third sessions, than in the first session. The detection probability over the 3 days was significantly higher in both the second and third sessions, than in the first session $(P<$ $1.1 \times 10^{-6}$ and $P<2.0 \times 10^{-7}$, respectively). The detection probability of the suddenly appearing disc remained high over the 3 days. These results indicate that the detection probability of the slowly appearing disc showed behavioral STI within a few minutes between the first and second sessions. This behavioral STI had mostly disappeared by the next day. However, the detection probability of the slowly appearing disc in the first session showed a slight increase over the 3 days, suggesting that behavioral LTI of detection probability could be induced under some circumstances. As for the control left visual field, the two visual stimuli were noticed with no failures throughout the 3 days.

\section{Behavioral LTI of Visual Stimulus Detection}

Three days after the behavioral STI experiment, the slowly appearing disc was presented in the impaired right visual field for 10 trials to begin the research on behavioral LTI induction. Unexpectedly, the stimulus was noticed in 8 out of 10 trials in the first session, and in 10 out of 10 trials in the second and third sessions (Figure 3C, Supplementary Table 1). In the first sessions over consecutive 4 days, the slowly appearing disc was noticed in 34 trials and not in 6 trials. This detection probability was significantly higher than that of the results shown in Figure 3A (detected in 7 trials and not in 23 trials, $P<5.9$ $\left.\times 10^{-8}\right)$. These results indicate that behavioral LTI of visual stimulus detection was induced in the impaired right visual field of the patient.

\section{Visual Perception After Behavioral LTI}

We estimated the extent of visual perception after behavioral LTI. We presented moving gratings in the impaired right visual field and randomly moved to the right or left (Supplementary Video 7). The patient correctly reported the direction of movement in all 50 trials (Figure 3D, Supplementary Table 1). The same experiment was performed in the control left visual field, and he again reported the correct direction of movement in all 50 trials.

Next, we presented a randomly selected Japanese Hiragana letter to the impaired right visual field for $0.5 \mathrm{~s}$ (Supplementary Video 8), and asked the patient to read the letter. Of the 50 trials, he correctly read in 21 trials, incorrectly in 11 trials, and detection failure was observed in 18 trials 

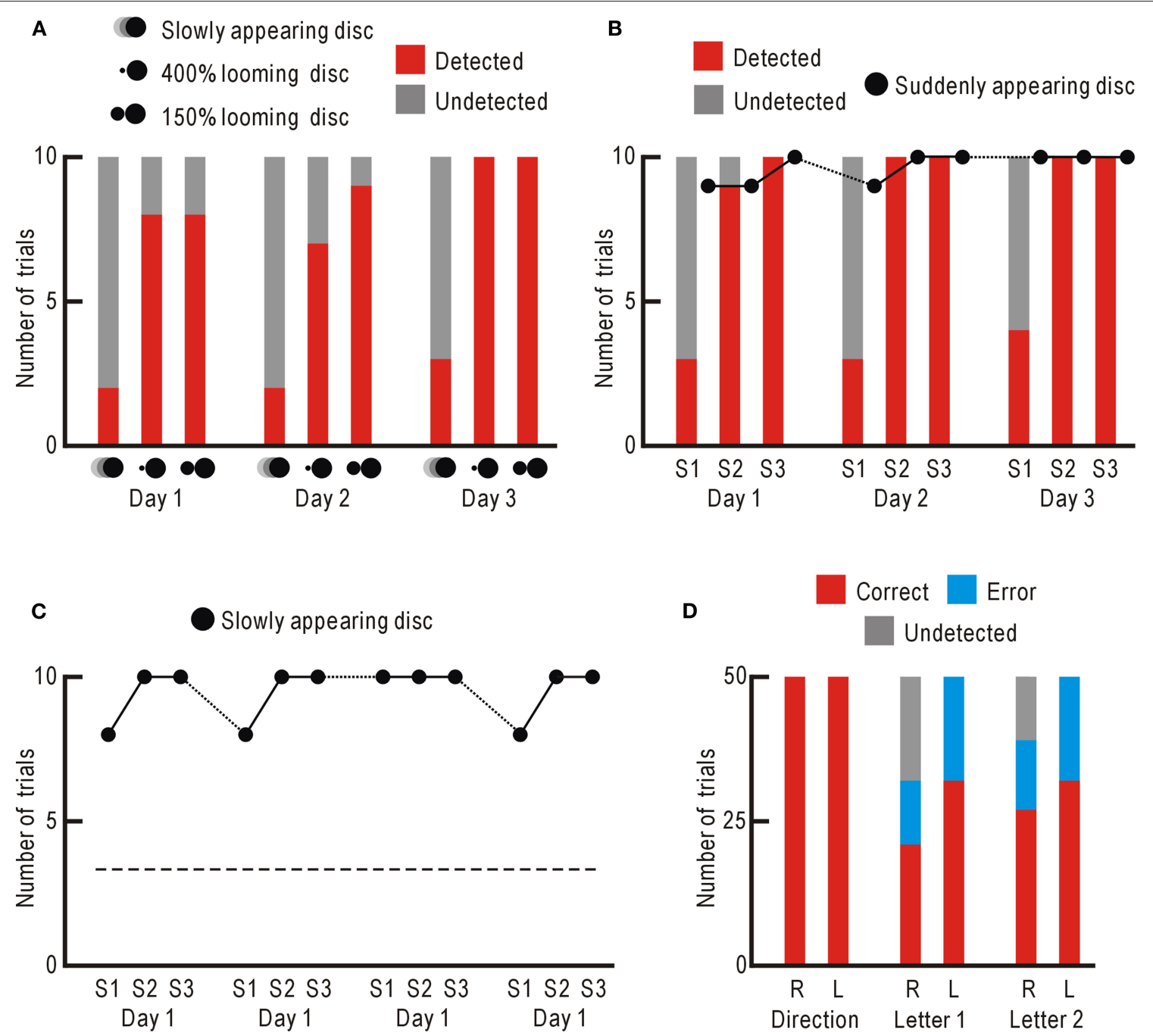

FIGURE 3 | (A) Stimulus detection ability in the impaired right visual field. Trials with successful detections are shown in red, and those with failures are shown in gray. (B) Behavioral STI of slowly appearing disk detection. For the slowly appearing disc, trials with successful detections are shown in red, and those with failures are shown in gray. For the suddenly appearing disc, only the numbers of trials with successful detections are shown with black dots. (C) Behavioral LTI of slowly appearing disk detection. Only the numbers of trials with successful detections are shown with black dots. The averaged detection probability of the slowly appearing disc in (A) are shown with broken line. (D) Visual perception after behavioral LTI in the impaired right visual field (R) and control left visual field (L). Trials with correct reports are shown in red, those with error reports are in blue, and those with detection failures are in gray.

(Figure 3D, Supplementary Table 1). As for the trials with successful detection, the correct answer rate $(65.6 \%)$ was significantly higher than that of random choice $(2.2 \%, P<5.7$ $\left.\times 10^{-7}\right)$. When the letters were presented in the control left visual field, he correctly read in 32 trials, incorrectly in 18 trials, and no detection failure was observed. The correct answer rate (64.0\%) was comparable to the value of $65.6 \%$ in the impaired right visual field. The same experiment was repeated the next day. The correct answer rate (69.2\%) in the impaired right visual field was significantly higher that of random choice $(P<3.6 \times$ $\left.10^{-9}\right)$, and again comparable to that in the control left visual field (64.0\%). This patient could not report presentation of static visual stimuli (e.g., static discs, Supplementary Video 1) in the impaired right visual field before behavioral STI and LTI of visual stimulus detection, and therefore, it is very unlikely that he could read letters presented in the impaired right visual field before behavioral STI and LTI.

\section{DISCUSSION}

Alarming visual stimuli, such as looming or suddenly appearing black shadows, suggest the approach of a potential predator and thus are preferentially detected to trigger escape behavior, even in primitive animals with undeveloped visual cortex $(27,28)$. In primates including humans, looming stimuli are also strongly recognized $(23,24)$, possibly because the stimuli suggest the presence of rapidly moving objects that may collide with the face. These stimuli could be perceived via the visual information 
mediated by the SC and amygdala $(8-10,27)$ or direct thalamic afferents to higher visual cortices $(29,30)$, since alarming visual stimuli or salient and dynamic visual stimuli presented in the impaired right visual field were sometimes noticed by the patient, as reported previously (26). The present case is characterized by a series of behavioral STI induction within a few minutes, followed by behavioral LTI persisting for more than a few days, in detection ability of visual stimuli. The time course of functional changes suggests that they are produced by some neural plasticity with similar time course, such as synaptic short-term potentiation (STP) and subsequent synaptic long-term potentiation (LTP) of neural circuits $(31,32)$. Although many studies have shown positive effects of rehabilitation for cortical blindness (3-5), training is expensive and takes a long time. Furthermore, it may be ineffective for some patients. Therefore, a simple and easy to perform screening test to determine the probability of recovery from cortical blindness is needed. Our behavioral STI experiment, that can be completed within $10 \mathrm{~min}$, may serve as a clinical screening test for anticipating recovery from cortical blindness.

The letter perception experiment shown in Figure 3D strongly suggests that functional recovery is restricted only to the detection ability of visual stimuli in the impaired visual field. Once detected, analysis of visual stimuli was performed with the same accuracy as when the stimulus was presented to the control visual field. These results are well explained by the assumption that neural circuits between neurons involved in blindsight and those involved in conscious sight show repeated synaptic STP followed by synaptic LTP. Synaptic STP and LTP are induced when presynaptic and postsynaptic neurons are activated simultaneously $(33,34)$. Presentation of alarming visual stimuli activates not only presynaptic neurons involved in blindsight but also postsynaptic neurons involved in conscious sight by changes in arousal level, which are also produced by alarming visual stimuli via the SC and amygdala $(8-10,27)$. After synaptic LTP has been established, visual information obtained through the SC becomes available for conscious sight neurons, and blindsight can be rapidly converted to conscious sight as a result.

Visual information obtained through the V1 is processed by two different pathways: the dorsal and ventral streams (35). The dorsal stream mainly analyzes movements and spatial information included in visual stimuli ("where" pathway), whereas the ventral stream is important for processing shape and texture of visual stimuli ("what" pathway). The ventral stream extends to the temporal cortex, and contains a group of neurons responding to a specific category of visual objects $(36,37)$. Visual information mediated via the SC projects to the higher visual cortices of the dorsal stream (11-14). The excellent perception of moving grating direction in the impaired right visual field (Figure 3D) may be explained as a function of this projection. The SC is also known to project to the amygdala, and this pathway seems to process affective shape information such as shadows of predators (11) or expressions on the face $(9,11)$. However, the perception of Japanese Hiragana letters in the impaired right visual field (Figure 3D) is unlikely attributed to functions of the amygdala. Recently, the presence of other visual pathways from the SC to the postrhinal cortex (38), and from the postrhinal cortex to the ectorhinal cortex and surrounding areas (39) has been identified in rodents. Since the ectorhinal cortex is located ventral to the auditory cortex, it probably corresponds to one of the higher visual cortices of the ventral stream in primates (35). The reasonable perception of Japanese Hiragana letters in the impaired right visual field (Figure 3D) may be explained as a function of human analogs to these murine pathways. The remaining question is which entities of the neural circuits exhibit neural plasticity responsible for the behavioral changes. Possibly, the potentiated synaptic circuits may be dispersed within higher visual cortices that are involved in both blindsight and conscious sight. It has been suggested that visual afferent pathways, which bypass the V1 and directly target higher visual cortices, are strengthened in patients with blindsight (40-43). Activity-dependent changes in these pathways are also likely candidates to explain the present findings. Obviously, however, the present results are far from sufficient to elucidate the underlying mechanisms, and various discussion in the present case report should be tested by further experimental studies on multiple cases of cortical blindness.

\section{DATA AVAILABILITY STATEMENT}

All datasets for this study are included in the article/Supplementary Material.

\section{ETHICS STATEMENT}

The protocol of this study was approved by the Local Ethics Committee of Kashiwazaki General Hospital and Medical Center (2019-05-21). This study was carried out in accordance with the recommendations of the Local Ethics Committee and the Declaration of Helsinki, with the written informed consent of the patient for the publication of this case report.

\section{AUTHOR CONTRIBUTIONS}

KS mainly performed experiments. IW and MT helped with experiments. TF and SH helped with data analyses. KS mainly wrote the manuscript. All authors discussed the results and edited the manuscript.

\section{FUNDING}

This work was supported by Grant-in-Aid for Scientific Research (No. 16H01892) to KS.

\section{ACKNOWLEDGMENTS}

We thank the patient for participating in this study.

\section{SUPPLEMENTARY MATERIAL}

The Supplementary Material for this article can be found online at: https:/www.frontiersin.org/articles/10.3389/fneur. 2020.00069/full\#supplementary-material 


\section{REFERENCES}

1. Breitmeyer BG. Contributions of magno- and parvocellular channels to conscious and non-conscious vision. Philos Trans R Soc Lond B. (2014) 369:20130213. doi: 10.1098/rstb.2013.0213

2. Hurme M, Koivisto M, Revonsuo A, Railo $H$. Early processing in primary visual cortex is necessary for conscious and unconscious vision while late processing is necessary only for conscious vision in neurologically healthy humans. Neuroimage. (2017) 150:230-8. doi: 10.1016/j.neuroimage.2017.02.060

3. Perez C, Chokron S. Rehabilitation of homonymous hemianopia: insight into blindsight. Front Integr Neurosci. (2014) 8:82. doi: 10.3389/fnint.2014. 00082

4. Frolov A, Feuerstein J, Subramanian PS. Homonymous hemianopia and vision restoration therapy. Neurol Clin. (2017) 35:29-43. doi: 10.1016/j.ncl.2016.08.010

5. Hadid V, Lepore F. From cortical blindness to conscious visual perception: theories on neuronal networks and visual training strategies. Front Syst Neurosci. (2017) 11:64. doi: 10.3389/fnsys.2017. 00064

6. Poppel E, Held R, Frost D. Leter: residual visual function after brain wounds involving the central visual pathways in man. Nature. (1973) 243:295-6. doi: $10.1038 / 243295 \mathrm{a} 0$

7. Weiskrantz L, Warrington EK, Sanders MD, Marshall J. Visual capacity in the hemianopic field following a restricted occipital ablation. Brain. (1974) 97:709-28. doi: 10.1093/brain/97.1.709

8. Vuilleumier P, Armony JL, Driver J, Dolan RJ. Distinct spatial frequency sensitivities for processing faces and emotional expressions. Nat Neurosci. (2003) 6:624-31. doi: 10.1038/nn1057

9. Tamietto M, Pullens P, de Gelder B, Weiskrantz L, Goebel R. Subcortical connections to human amygdala and changes following destruction of the visual cortex. Curr Biol. (2012) 22:1449-55. doi: 10.1016/j.cub.2012. 06.006

10. Méndez-Bértolo C, Moratti S, Toledano R, Lopez-Sosa F, Martínez-Alvarez R, Mah YH, et al. A fast pathway for fear in human amygdala. Nat Neurosci. (2016) 19:1041-9. doi: 10.1038/nn.4324

11. Lyon DC, Nassi JJ, Callaway EM. A disynaptic relay from superior colliculus to dorsal stream visual cortex in macaque monkey. Neuron. (2010) 65:270-9. doi: 10.1016/j.neuron.2010.01.003

12. Tohmi M, Meguro R, Tsukano H, Hishida R, Shibuki K. The extrageniculate visual pathway generates distinct response properties in the higher visual areas of mice. Curr Biol. (2014) 24:587-97. doi: 10.1016/j.cub.2014. 01.061

13. Yoshida M, Hafed ZM, Isa T. Informative Cues Facilitate Saccadic Localization in Blindsight Monkeys. Front Syst Neurosci. (2017) 11:5. doi: 10.3389/fnsys.2017.00005

14. Kinoshita M, Kato R, Isa $K$, Kobayashi $K$, Kobayashi $K$, Onoe H, et al. Dissecting the circuit for blindsight to reveal the critical role of pulvinar and superior colliculus. Nat Commun. (2019) 10:135. doi: 10.1038/s41467-018-08058-0

15. Zhang X, Kedar S, Lynn MJ, Newman NJ, Biousse V. Natural history of homonymous hemianopia. Neurology. (2006) 66:901-5. doi: 10.1212/01.wnl.0000203338.54323.22

16. Sahraie A, Trevethan CT, MacLeod MJ, Murray AD, Olson JA, Weiskrantz L. Increased sensitivity after repeated stimulation of residual spatial channels in blindsight. Proc Natl Acad Sci USA. (2006) 103:14971-6. doi: 10.1073/pnas.0607073103

17. Das A, Tadin D, Huxlin KR. Beyond blindsight: properties of visual relearning in cortically blind fields. J Neurosci. (2014) 34:11652-64. doi: 10.1523/JNEUROSCI.1076-14.2014

18. Marshall RS, Ferrera JJ, Barnes A, Xian Zhang, O’Brien KA, Chmayssani $\mathrm{M}$, et al. Brain activity associated with stimulation therapy of the visual borderzone in hemianopic stroke patients. Neurorehabil Neural Repair. (2008) 22:136-44. doi: 10.1177/1545968307305522

19. Bridge H, Thomas O, Jbabdi S, Cowey A. Changes in connectivity after visual cortical brain damage underlie altered visual function. Brain. (2008) 131:1433-44. doi: 10.1093/brain/awn063
20. Ajina S, Kennard C, Rees G, Bridge H. Motion area V5/MT+ response to global motion in the absence of V1 resembles early visual cortex. Brain. (2015) 138:164-78. doi: 10.1093/brain/awu328

21. Melloni L, Molina C, Pena M, Torres D, Singer W, Rodriguez E. Synchronization of neural activity across cortical areas correlates with conscious perception. J Neurosci. (2007) 27:2858-65. doi: 10.1523/ JNEUROSCI.4623-06.2007

22. Silvanto J. Why is "blindsight" blind? A new perspective on primary visual cortex, recurrent activity and visual awareness. Conscious Cogn. (2015) 32:1532. doi: 10.1016/j.concog.2014.08.001

23. Schiff W, Caviness JA, Gibson JJ. Persistent fear responses in rhesus monkeys to the optical stimulus of "looming". Science. (1962) 136:982-3. doi: $10.1126 /$ science.136.3520.982

24. Lin JY, Murray SO, Boynton GM. Capture of attention to threatening stimuli without perceptual awareness. Curr Biol. (2009) 19:1118-22. doi: 10.1016/j.cub.2009.05.021

25. Kanda Y. Investigation of the freely available easy-to-use software 'EZR' for medical statistics. Bone Marrow Transplant. (2013) 4:452-8. doi: 10.1038/bmt.2012.244

26. Zeki S, Ffytche DH. The Riddoch syndrome: insights into the neurobiology of conscious vision. Brain. (1998) 121:25-45. doi: 10.1093/brain/12 1.1 .25

27. Fotowat H, Gabbiani F. Collision detection as a model for sensory-motor integration. Annu. Rev. Neurosci. (2011) 34:1-19. doi: 10.1146/annurev-neuro-061010-113632

28. Temizer I, Donovan JC, Baier H, Semmelhack JL. A visual pathway for looming-evoked escape in larval zebrafish. Curr Biol. (2015) 25:1823-34. doi: 10.1016/j.cub.2015.06.002

29. Schmid MC, Mrowka SW, Turchi J, Saunders RC, Wilke M, Peters AJ, et al. Blindsight depends on the lateral geniculate nucleus. Nature. (2010) 466:373-7. doi: 10.1038/nature09179

30. Ajina S, Pestilli F, Rokem A, Kennard C, Bridge H. Human blindsight is mediated by an intact geniculo-extrastriate pathway. eLife. (2015) 4:e08935. doi: 10.7554/eLife.08935

31. Volianskis A, Bannister N, Collett VJ, Irvine MW, Monaghan DT, Fitzjohn $\mathrm{SM}$, et al. Different NMDA receptor subtypes mediate induction of long-term potentiation and two forms potentiation at CA1 synapses in rat hippocampus in vitro. J Physiol. (2013) 591:955-72. doi: 10.1113/jphysiol.2012.247296

32. Park P, Volianskis A, Sanderson TM, Bortolotto ZA, Jane DE, Zhuo M, et al. NMDA receptor-dependent long-term potentiation comprises a family of temporally overlapping forms plasticity that are induced by different patterns of stimulation. Philos Trans R Soc Lond B. (2013) 369:20130131. doi: $10.1098 /$ rstb.2013.0131

33. Bliss TV, Collingridge GL. A synaptic model of memory: longterm potentiation in the hippocampus. Nature. (1993) 361:31-9. doi: 10.1038/361031a0

34. Feldman DE. The spike-timing dependence of plasticity. Neuron. (2012) 75:556-71. doi: 10.1016/j.neuron.2012.08.001

35. Rizzolatti RG, Matelli M. Two different streams form the dorsal visual system: anatomy and functions. Exp Brain Res. (2003) 153:146-57. doi: 10.1007/s00221-003-1588-0

36. Kiani R, Esteky H, Mirpour K, Tanaka K. Object category structure in response patterns of neuronal population in monkey inferior temporal cortex. J Neurophysiol. (2007) 97:4296-309. doi: 10.1152/jn.00024.2007

37. Kriegeskorte N, Mur M, Ruff DA, Kiani R, Bodurka J, Esteky H, et al. Matching categorical object representations in inferior temporal cortex of man and monkey. Neuron. (2008) 60:1126-41. doi: 10.1016/j.neuron.2008. 10.043

38. Beltramo R, Scanziani M. A collicular visual cortex: Neocortical space for an ancient midbrain visual structure. Science. (2019) 363:64-69. doi: 10.1126/science.aau7052

39. Nishio N, Tsukano H, Hishida R, Abe M, Nakai J, Kawamura M, et al. Higher visual responses in the temporal cortex of mice. Sci Rep. (2018) 8:11136. doi: 10.1038/s41598-018-29530-3

40. Leh SE, Johansen-Berg H, Ptito A. Unconscious vision: new insights into the neuronal correlate of blindsight using diffusion tractography. Brain. (2006) 129:1822-32. doi: 10.1093/brain/awl111 
41. Leh SE, Mullen KT, Ptito A. Absence of S-cone input in human blindsight following hemispherectomy. Eur $J$ Neurosci. (2006) 24:2954-60. doi: 10.1111/j.1460-9568.2006.0 5178.x

42. Bridge H, Leopold DA, Bourne JA. Adaptive pulvinar circuitry supports visual cognition. Trends Cogn Sci. (2016) 20:146-57. doi: 10.1016/j.tics.2015. 10.003

43. Hurme M, Koivisto M, Revonsuo A, Railo H. V1 activity during feedforward and early feedback processing is necessary for both conscious and unconscious motion perception. Neuroimage. (2019) 185:313-21. doi: 10.1016/j.neuroimage.2018.10.058
Conflict of Interest: The authors declare that the research was conducted in the absence of any commercial or financial relationships that could be construed as a potential conflict of interest.

Copyright (c) 2020 Shibuki, Wakui, Fujimura, Tomikawa and Hasegawa. This is an open-access article distributed under the terms of the Creative Commons Attribution License (CC BY). The use, distribution or reproduction in other forums is permitted, provided the original author(s) and the copyright owner(s) are credited and that the original publication in this journal is cited, in accordance with accepted academic practice. No use, distribution or reproduction is permitted which does not comply with these terms. 